\title{
O luto na agenda das equipes multiprofissionais de oncologia e cuidados paliativos: apresentação de um Programa de Assistência a Familiares na Universidade Federal de São Paulo
}

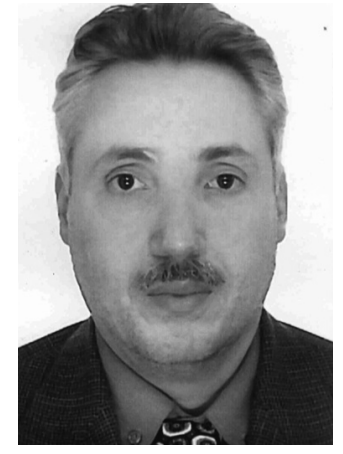

João Paulo

Consentino

Departamento de Medicina e Projeto de Proteção ao Luto - Universidade Federal de São Paulo (Unifesp), São Paulo, Brasil joaopaulocsolano@uol.com.br contato@casadahumanidade. org.br

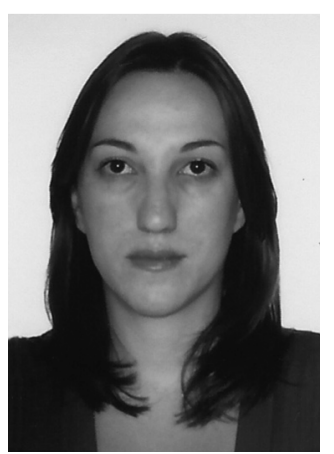

\section{Marcela Alice}

Bianco

Projeto de Proteção ao Luto - Universidade Federal de São Paulo (Unifesp), São Paulo, Brasil

\section{Renata Moraes Ferreira}

Projeto de Proteção ao Luto - Universidade Federal de São Paulo, São Paulo, Brasil

\section{Resumo}

Nos últimos anos tem sido chamada a atenção para o fato de que o conceito de cuidados paliativos deve ser ampliado e de que as reações de luto dos familiares dos pacientes sejam incluídas como foco de atenção por equipes multiprofissionais. Nos hospitais, o luto afeta o paciente que recebe a notícia de ter um prognóstico reservado, mas também afeta seus familiares e as equipes técnicas que do paciente se acercam. Pelo seu potencial de complicar-se e causar prejuízos físicos, psíquicos e comunitários, o luto já foi referido como um problema de saúde pública, capaz de onerar as redes assistenciais de forma silenciosa. O presente trabalho tem por objetivos localizar o problema do luto nas equipes hospitalares de cuidados paliativos e de oncologia, e apresentar um programa de assistência grupal a familiares enlutados que tem contribuído para a melhoria da qualidade da comunicação e veiculação de informações em um hospital-escola de São Paulo.

\section{Palavras-chave}

Luto, cuidados paliativos, oncologia, assistência multiprofissional, terminalidade 
O ano de 2002 poderá ser lembrado como um divisor de águas na história do desenvolvimento do paliativismo no mundo todo. Isto porque, em 2002, documento emitido pela Organização Mundial da Saúde (OMS) cunhou um novo conceito para Cuidados Paliativos, que veio a substituir o conceito anterior de 1990 . O novo conceito, mais abrangente, pode ser resumido ressaltando-se seus aspectos nucleares: cuidados paliativos passaram a ser entendidos como uma abordagem que visa melhorar a qualidade de vida de pacientes e de seus familiares ao enfrentarem doença crônica, progressivamente debilitante e fatal, através da prevenção e alívio dos sofrimentos a ela inerentes, por meio do diagnóstico precoce e impecáveis avaliação e tratamento da dor e de outros problemas físicos, psicossociais e espirituais (SEPULVEDA et al., 2002; WORLD HEALTH ORGANIZATION, 2002; 2004).

A leitura dos documentos da OMS permite depreender que o alargamento do conceito de Cuidados Paliativos se deu em quatro dimensões. Em primeiro lugar, não apenas os pacientes terminais são os elegíveis para receber cuidados paliativos, mas todos os pacientes com doenças crônico-degenerativas, progressivamente debilitantes e fatais. Isto significa que tanto pacientes com uma neoplasia fora de possibilidades terapêuticas, como aqueles com síndrome de imunodeficiência humana adquirida (Aids), com falência cardíaca, doença pulmonar obstrutiva crônica, insuficiência renal crônica, doenças neurodegenerativas, os seqüelados de traumas craniencefálicos e acidentes vasculares cerebrais, pacientes com fibrose cística, entre outros, passam a ser alvo dos cuidados paliativos - e já a partir do diagnóstico. Em segundo lugar, ficou determinado que não só às necessidades físico-sintomatológicas dos doentes, mas também às suas demandas psíquicas, sociais $e$ espirituais deve ser dada atenção. Em terceiro lugar, tanto o paciente como sua família passam a ser alvo do planejamento e do benefício da assistência. Em quarto lugar, o luto dos familiares passa a ser foco de atenção e assistência, tanto antes como depois da morte do paciente.

O luto deve ser definido como um conjunto de reações emocionais, físicas, comportamentais e sociais que aparecem como resposta a uma perda - seja uma perda real ou fantasiosa (um "medo de perder"), seja uma perda por morte ou pela cessação/diminuição de uma função, possibilidade ou oportunidade (PARKES, 1998a).

Trabalhos conduzidos nos países mais desenvolvidos têm colocado as intervenções com enlutados como um importante problema de saúde pública, devido às implicações sobre a saúde geral dos sobreviventes (YOUNG et al., 1963; STROEBE et al., 1993; PARKES, 1998b; KATO, 1999). Conseqüências adversas à saúde física incluem prejuízo temporário da imunidade corporal, aumento no número de consultas médicas, hospitalizações, cirurgias e aumento da taxa de mortalidade das populações enlutadas, quando comparadas à população geral (YOUNG et al., 1963; STROEBE et al., 1993; PARKES, 1998b). Conseqüências à saúde mental têm sido descritas em termos de variados níveis de depressão, ansiedade, desespero, descrença e/ou "paralisia emocional” (BOWLBY, 1961; PARKES, 1998b).
Parece haver um atual consenso de que, entre os extremos entre uma reação de luto adaptada, autolimitada, não merecedora de intervenção terapêutica, de um lado, e as síndromes psiquiátricas de outro lado, há uma população intermediária de indivíduos que apresentarão uma síndrome de luto complicado - indivíduos que merecerão tratamento que os alivie de intensos e deletérios sintomas emocionais (PRIGERSON 1997; 1999; SHEAR, 2001).

$\mathrm{Na}$ área da saúde, caso sejam observadas as várias maneiras pelas quais as pessoas estão sendo afetadas pelo luto, muito poderá ser feito para a melhoria da comunicação entre pacientes, familiares e técnicos. Em hospitais gerais, temos que observar o luto que incide em pelo menos quatro situações (SOLANO, 2006):

1. luto do paciente, quando recebe a notícia de estar com alguma doença incurável, ou percebe a progressão inexorável de suas limitações e vê que a morte se aproxima;

2. luto da família ao acompanhar, portanto, o declínio funcional de um de seus membros;

3. luto dos sobreviventes (familiares e amigos) após a morte do paciente;

4. luto da equipe técnica que cuida do paciente.

No presente artigo, além de nos postarmos junto à OMS ao advogar que a assistência ao luto precisa estar incluída nas atividades dos paliativistas e oncologistas, iremos apresentar um programa de apoio a enlutados que se originou dos contatos com os familiares dos pacientes que vimos atendendo no Setor de Cuidados Paliativos da Universidade Federal de São Paulo (UNIFESP).

A experiência de trabalhar em equipe multiprofissional de Cuidados Paliativos precocemente demonstrou que urgia implantarmos um programa de apoio em saúde mental aos familiares e cuidadores dos pacientes assistidos pelo setor. Tal programa foi fundado em outubro de 2003 e recebeu o nome de PROLU - Projeto de Proteção ao Luto. A idéia foi acolhida pela Reitoria da Universidade, que disponibilizou um anfiteatro no Centro de Assistência e Ensino em Enfermagem (CAENF), localizado a 400 metros da Universidade. Cuidadores, familiares e amigos de todos os pacientes que eram encaminhados ao Setor de Cuidados Paliativos (na sua maioria portadores de câncer em estado avançado, inseridos em famílias com baixa renda e residentes em bairros periféricos de São Paulo) tornaram-se elegíveis de receber um convite para participar das atividades do PROLU. A essas pessoas foram oferecidas duas atividades assistenciais seqüenciais: (a) participar de um grupo onde se encontravam com outros familiares e cuidadores de pacientes fora de possibilidades terapêuticas de cura (grupo com cuidadores); e (b) participar, após a morte de seu ente querido, de um grupo com outros enlutados (grupo com enlutados). Os objetivos das atividades (a) e (b) são mostrados no Quadro 1.

Um cuidador ou familiar que freqüentasse a atividade (a) era instruído quanto ao fato de que, após a morte de seu enfermo, seria convidado a participar do grupo com outros enlutados (atividade b). No entanto, o grupo 
com enlutados também era alimentado por clientes que nunca tinham participado da atividade a, pois, após cerca de duas semanas do óbito de um paciente, uma carta de condolências era enviada e pelo menos um telefonema era feito à família. $\mathrm{O}$ quadro 2 apresenta o modelo de carta de condolências adotado pelo PROLU.

\section{Quadro 1 - Objetivos das atividades do PROLU - Projeto de Proteção ao Luto da Universidade Federal de São Paulo}

\author{
(a) Grupo com cuidadores \\ Treinar pessoal não-especializado para as- \\ sistência domiciliar a enfermos com alto grau \\ de dependência, familiares ou pertencentes à \\ comunidade.
}

\section{(b) Grupo com enlutados}

Favorecer que familiares de pacientes que tinham morrido aos cuidados do hospital da universidade (Hospital São Paulo) se encontrassem e trocassem as experiências de suas dores.

Manter um vínculo de alguns meses entre os familiares e a instituição.

\section{Acompanhar o processo de enlutamento dos} familiares, selecionando os casos mais graves e os encaminhando para tratamento específico.

Treinar líderes de grupos de auto-ajuda para a comunidade.

Cada um dos grupos acontecia quinzenalmente, era verbal aberto, tinha duração de 90 minutos, no máximo 12 participantes e era conduzido por um terapeuta principal (psiquiatra) e um terapeuta observador (psicóloga ou assistente social). A freqüência aos grupos era espontânea e não havia limite quanto ao número de pessoas que cada família podia trazer; era permitida a entrada de amigos da família; pessoas com menos de 16 anos não eram convidadas para freqüentar o grupo, mas não eram impedidas de entrar caso comparecessem.

A técnica utilizada na condução de cada um dos grupos diferia. O grupo de cuidadores (a) abrigava formato psicopedagógico, com entrega de informações práticas que ajudassem a cuidar de um enfermo em casa. Já o grupo com enlutados (b) objetivava oferecer apoio psicoterápico, sendo o estudo do processo de enlutamento ativamente buscado e os participantes estimulados a investirem em seu trabalho de luto; os casos de luto complicado eram identificados pelo terapeuta principal e abordados com medidas adicionais (farmacoterapia ou encaminhamento para serviço de saúde mental, onde vínculos de terapia individual pudessem ser constituídos).

\section{Quadro 2 - Modelo de Carta de Condolências adotado pelo PROLU $\left({ }^{*}\right)$}

\begin{abstract}
São Paulo, _ de __ de
À família e aos amigos de

A equipe de Cuidados Paliativos desta Instituição espera que este momento de pesar que ora vocês estão sofrendo passe o mais breve possível. Queremos agradecer pela confiança que vocês demonstraram ao entregarem um familiar amado seu aos nossos cuidados, e ao permitirem que adentrássemos a intimidade de seu lar. Principalmente, queremos parabenizá-los por terem cuidado de até os seus últimos dias. Sabemos que a batalha foi árdua, e vimos de perto com que coragem e dedicação vocês lutaram. Agora, passada a luta, esperamos que todos nós possamos descansar. Sabemos que, mesmo descansando, a imagem do(a)

vivo(a) não será esquecida. E sabemos que a força que herdamos dele(a) nos ajudará a enfrentar as próximas batalhas junto a nossos semelhantes. Obrigado, e guardem nossos telefones, pois queremos continuar à disposição de vocês.
\end{abstract}

(Assinatura)

Setor de Cuidados Paliativos do Departamento de Medicina Universidade Federal de São Paulo

(*) A carta é enviada somente após duas semanas do óbito do paciente e se acompanha de (pelo menos) um contato telefônico no qual se convida a família para conhecer o grupo com enlutados (atividade b).

A atividade (a) falhou em constituir-se porque um grupo de trabalho não foi formado ao longo do tempo. Nenhuma reunião contou com um número de participantes superior a três, e houve várias para as quais não houve presença. A freqüência altamente irregular dos poucos participantes inviabilizou qualquer tentativa de mensuração de resultados.

A atividade (b) tem acontecido desde o início do projeto (outubro/2003), com uma interrupção entre junho e outubro de 2004, por motivo de viagem do terapeuta principal, e outra entre janeiro e outubro de 2006, para reforma de um novo espaço. Além dos encontros quinzenais, cinco "eventos sociais" foram promovidos (quatro comemorações de Natal e uma "despedida" do terapeuta), com grande comparecimento de ex-participantes. Até o momento, 56 enlutados foram recebidos nos grupos. Seis casos de luto complicado (11\%) foram identificados e foram ou estão sendo tratados. O quadro 3 mostra os critérios diagnósticos utilizados no PROLU para identificar casos de luto complicado (ou patológico), uma categoria nosográfica cuja inclusão na próxima ver- 
são (quinta) do Diagnostic and Statistical Manual for Mental Disorders (DSM-V) está sendo cogitada (PRIGERSON 1999). A partir de dezembro de 2004, o serviço se tornou disponível para quaisquer pacientes da UNIFESP e para o público em geral.

Famílias enlutadas têm freqüentemente se mostrado confortadas pela carta de condolências que recebem. Nenhuma mensuração objetiva do impacto deste procedimento foi feita até o momento, porém.

\section{Quadro 3 - Proposta de PRIGERSON et al. para inclusão da categoria de Luto Complicado no DSM-V}

\author{
Critério A: perda de um ser amado por morte, \\ seguida de reação com pelo menos três dos \\ seguintes: \\ - pensamentos intrusivos sobre o morto; \\ - ânsia pelo reencontro; \\ - comportamento de busca pelo morto; \\ - vivência de solidão como resultado da morte.
}

Critério B: pelo menos quatro dos seguintes: - vivência de falta de objetivos ou valoração do futuro como fútil;

- vivência subjetiva de anestesia emocional, desligamento do mundo ou de perda da responsividade emocional;

- dificuldade para aceitar a perda;

- sentimento de vida vazia ou sem sentido;

- sentimento de ter perdido parte de si mesmo

("morreu junto");

- colapso da visão de mundo;

- repetição de sintomas (ou hábitos de risco) do morto;

- excessiva irritabilidade, amargura ou raiva em relação ao ocorrido.

Critério C: pelo menos dois meses de duração.

Critério D: o distúrbio causa significativo prejuízo no funcionamento social, ocupacional ou em outras áreas importantes da vida do indivíduo.

Fonte: Adaptado de SOLANO 2006.

Tradução: João Paulo Consentino Solano

Impõe-se perguntar por que houve falha na intervenção (a). É pertinente levantar a hipótese de que famílias de baixa renda, moradoras de regiões longínquas na periferia de uma grande metrópole e que estão tentando cuidar de seu enfermo em casa (como é solicitado pela equipe de Cuidados Paliativos) encontrem dificuldade para deslocar um familiar/cuidador quinzenalmente ao hospital - pois isto poderia deixar o enfermo, por meio período do dia, sem cuidador e causar um acréscimo à sobrecarga emocional e econômica a que tais famílias já estão expostas.

O grupo com enlutados (atividade b) constituiu-se de pessoas que tinham perdido um ente querido e que foram convidadas para o grupo pelo mesmo profissional que tinha dado assistência domiciliar ao enfermo, um dos autores deste artigo. Esses sujeitos sabiam que este profissional seria o mesmo que conduziria o grupo com enlutados. Ao todo, 38 famílias receberam o convite para vir ao grupo. Vinte e sete famílias atenderam (taxa de resposta de $70 \%$ ), gerando a freqüência aos grupos de 56 sujeitos enlutados.

Entre junho e setembro de 2004, o grupo interrompeu suas atividades por motivo de força maior, e ficou claro que a partir de outubro de 2004 uma segunda fase na história do grupo se iniciara: a maior parte dos enlutados não voltaria para a continuação do trabalho, a despeito de terem sido contatados por telefone e correio. Após considerável ponderação entre os terapeutas, entendeu-se que não se deveria insistir nos convites, já que isto poderia significar a emissão de mensagens ambíguas quanto à capacidade desses ex-clientes cuidarem de seus trabalhos de luto - agora sem a ajuda do grupo. Esta segunda fase na história do grupo pode ter significado, na verdade e simplesmente, que um novo grupo se formou, pois houve renovação de quase todos os membros assíduos.

Grupos psicoterápicos com enlutados, em geral, podem seguir duas grandes linhas técnicas: uma que preza mais a expressão emocional dos membros e outra que privilegia as ações com que os membros devem modificar a realidade interna e externa para que seu trabalho de luto vá-se consolidando. Uma terceira opção técnica ao trabalhar com os grupos resulta da mescla das duas estratégias de abordagem. Esta é a técnica utilizada no PROLU, por achar-se que sua inerente flexibilidade pode acolher melhor as oscilações de demandas de um grupo aberto de psicoterapia (CONGRESSO INTERNACIONAL DE TANATOLOGIA E BIOÉTICA, 2005).

A opção de trabalhar com grupos de enlutados numa casa fora do hospital - embora casual a princípio - mostrou-se benéfica, pois muitas famílias que perderam entes queridos, após meses ou anos de acompanhamento nas várias enfermarias e ambulatórios do hospital, hesitariam, conforme sugerido na literatura especializada, em continuar freqüentando os mesmos espaços nas primeiras semanas ou meses de enlutamento (PARKES, 1998a; CONGRESSO INTERNACIONAL DE TANATOLOGIA E BIOÉTICA, 2005).

Desde dezembro de 2004, pessoas têm sido incluídas no grupo, ainda que não oriundas das famílias acompanhadas pelo Setor de Cuidados Paliativos. Até abril de 2005, 56 pessoas foram atendidas pelo PROLU, das quais oito tomaram conhecimento do serviço através da mídia.

Ao final do primeiro semestre de 2006, foi feita uma tentativa de mensurar o impacto da intervenção por meio de entrevistas aplicadas a todos os participantes e ex-participantes da atividade. Os resultados dessa avaliação serão apresentados em outro trabalho. 
Alguém já estimou que, para cada pessoa que morre, cinco parentes, amigos íntimos ou amores ficam de luto (ZISOOK, 2000). O que significa que qualquer pessoa envolvida em Oncologia e Cuidados Paliativos deve saber que encontrará muito mais reações de luto ao redor de seu paciente do que, propriamente, pacientes.

Trabalhar com questões relativas à morte e ao luto é ainda algo novo no Brasil, especialmente no meio hospitalar (e, principalmente, entre médicos). No entanto, é fora de dúvida que as recomendações emitidas pela OMS, quanto a estendermos os cuidados oferecidos aos pacientes terminais também a seus familiares e cuidadores, precisam ser implementadas entre nós.

Em seguida, conduziremos estudos de boa qualidade que mensurem a efetividade da intervenção, e com amostras maiores que aquela de que dispomos por ora. Temos podido observar, porém, mesmo antes de mensurar o impacto da intervenção, que ela é, por si só, capaz de refinar a qualidade das comunicações entre os enfermos e as equipes que trabalham no hospital, bem como prover a divulgação de informações críticas à prevenção de reações patológicas de luto na comunidade.

\section{Referências bibliográficas}

BOWLBY, J. Processes of mourning. The International Journal of Psycho-Analysis, v.42, n.4, p.317-340, 1961.

CONGRESSO INTERNACIONAL DE TANATOLOGIA E BIOÉTICA, 3, 2005, São Paulo. [nota: conferências proferidas por Dr. Colin Murray Parkes].

KATO, P.M.; MANN, T. A synthesis of psychological interventions for the bereaved. Clinical Psychology Review, 19, n. 3, p. 275-296, 1999.

PARKES, C.M. Bereavement. In: DOYLE, D.; HANKS, G.W.C.; MACDONALD, N. (Eds). Oxford Textbook of Palliative Medicine. $2^{\text {nd }}$ Ed. Oxford: Oxford University Press, 1998a. p 995-1010.

PARKES, C.M. Luto: estudos sobre a perda na vida adulta. São Paulo: Summus, 1998b. 290 p.
PRIGERSON, H.G.; BIERHALS, A. J.; KASL, S.V. et al.. Traumatic grief as a risk factor for mental and physical morbidity. American Journal of Psychiatry, v. 154, n. 5, p. 616-623, may 1997.

PRIGERSON, H.G.; SHEAR, M.K.; JACOBS, S.C. et al. Consensus criteria for traumatic grief: a preliminary empirical test. British Journal of Psychiatry, v.174, n.1, p.67-73, jan. 1999.

SEPULVEDA, C.; MARLIN, A.; YOSHIDA, T. et al.. Palliative Care: The World Health Organization's global perspective. Journal of Pain and Symptom Management, v. 24, n. 2, p. 91-96, aug. 2002.

SHEAR, M.K.; FRANK, E.; FOA, E. et al.. Traumatic grief treatment: a pilot study. American Journal of Psychiatry, v. 158, n. 9, p. 1506-1508, sep. 2001.

SOLANO, J. P. C. Luto. In: LOPES, A. C. (Ed). Diagnóstico e Tratamento. São Paulo: Manole, 2006. v.2, cap 16, p. 367-373.

STROEBE, M.S.; STROEBE, W. The mortality of bereavement: a review. In: STROEBE, M. S.; STROEBE, W.; HANSSON, R. O. (Eds). Handbook of bereavement. Cambridge: Cambridge University Press, 1993. p.175-207.

WORLD HEALTH ORGANIZATION. National cancer control programmes: policies and managerial guidelines, $2^{\text {nd }}$ ed. Geneva: World Health Organization, 2002. 203 p.

WORLD HEALTH ORGANIZATION. Palliative Care: the Solid Facts, Milan: World Health Organisation Regional Office for Europe, 2004. 32 p.

YOUNG, M.; BENJAMIN, B.; WALLIS, C. Mortality of widowers. Lancet, v.282, n.7305, p.454-457, aug. 1963.

ZISOOK'S. Understanding and Managing Bereavement in Palliative Care. In: CHOCHINOV, H.M.; BREITBART, W. (Eds). Handbook of Psychiatry in Palliative Medicine. Oxford: Oxford University Press, 2000. Chap.22, p.321-334. 


\section{Sobre os autores}

\section{João Paulo Consentino Solano}

Médico pela Faculdade de Ciências Médicas da Santa Casa de São Paulo (1987), psiquiatra pela Universidade Federal de São Paulo (UNIFESP, 1990), psicanalista pela Clínica "Roberto Azevedo" (2000-2005) e Research fellow em Cuidados Paliativos no Departamento de Cuidados Paliativos, Reabilitação e Política da Kings College London, Inglaterra (2004). É mestrando do Departamento de Medicina Preventiva da Faculdade de Medicina da Universidade de São Paulo (USP) e colaborador do Núcleo de Epidemiologia do Hospital Universitário (HUUSP). Foi médico do Setor de Cuidados Paliativos da UNIFESP (2000-2006). É professor da Disciplina de Clínica Médica do Departamento de Medicina e fundador-coordenador do Projeto de Proteção ao Luto da UNIFESP. Além disso, é fundador e presidente da ONG Casa da Humanidade e tem diversos artigos publicados em revistas internacionais como, por exemplo: Journal of Pain and Symptom Management, Hospice Information Bulletin, Psychiatry on Line.

\section{Marcela Alice Bianco}

Psicóloga pela Universidade Federal de São Carlos (2004) com especialização em Atendimento Multiprofissional Geriátrico e Gerontológico em Psicologia pelo Hospital Servidor Público Estadual (2006) e curso de extensão em Psicologia Hospitalar pelo Hospital do Coração (São Paulo). Com experiência em Psicologia Hospitalar, desenvolvendo ações preventivas e terapêuticas em saúde de adultos, idosos e seus familiares. Sua experiência inclui ainda: Psicologia Clínica, Psicoterapia Breve, Orientação Familiar e Coordenação de Grupos Informativos e de Treinamento na área da saúde. Recebeu recentemente uma menção honrosa no Prêmio de Incentivo em Ciência e Tecnologia para o SUS, do Departamento de Ciência e Tecnologia (DECIT) do Ministério da Saúde, na categoria Especialização, pela pesquisa "Aspectos psíquicos da relação paciente-família-equipe interdisciplinar em enfermaria geriátrica: impacto sobre o tratamento do idoso". 